\title{
Induction of Expandable Adipose-Derived Mesenchymal Stem Cells from Aged Mesenchymal Stem Cells by a Synthetic Self-Replicating RNA
}

\author{
Chika Miyagi-Shiohira ${ }^{1}$ D , Yoshiki Nakashima ${ }^{1}$, Naoya Kobayashi ${ }^{2}$, Shinji Kitamura ${ }^{3}$, \\ Issei Saitoh ${ }^{4}$, Masami Watanabe ${ }^{5}$ and Hirofumi Noguchi ${ }^{1, *}$ (D) \\ 1 Department of Regenerative Medicine, Graduate School of Medicine, University of the Ryukyus, \\ 207 Uehara, Nishihara, Okinawa 903-0215, Japan; chika@med.u-ryukyu.ac.jp (C.M.-S.); \\ nakasima@med.u-ryukyu.ac.jp (Y.N.) \\ 2 Department of Surgery, Okayama Saidaiji Hospital, Okayama 704-8192, Japan; n-kobayashi@saidaiji-hp.or.jp \\ 3 Department of Nephrology, Rheumatology, Endocrinology and Metabolism, Okayama University Graduate \\ School of Medicine, Dentistry and Pharmaceutical Sciences, Okayama 700-8558, Japan; \\ kitamura@okayama-u.ac.jp \\ 4 Division of Pediatric Dentistry, Graduate School of Medical and Dental Science, Niigata University, \\ Niigata 951-8514, Japan; isaito@dent.niigata-u.ac.jp \\ 5 Department of Urology, Okayama University Graduate School of Medicine, Dentistry and Pharmaceutical \\ Sciences, Okayama 700-8558, Japan; masami5@md.okayama-u.ac.jp \\ * Correspondence: noguchih@med.u-ryukyu.ac.jp; Tel.: +81-98-895-1696; Fax: +81-98-895-1684
}

Received: 3 September 2018; Accepted: 1 November 2018; Published: 6 November 2018

\begin{abstract}
Adipose-derived mesenchymal stem cells (ADSCs) have attracted attention due to their potential for use in the treatment of various diseases. However, the self-renewal capacity of ADSCs is restricted and their function diminishes during passage. We previously generated induced tissue-specific stem cells from mouse pancreatic cells using a single synthetic self-replicating Venezuelan Equine Encephalitis (VEE)-reprogramming factor (RF) RNA replicon (SR-RNA) expressing the reprogramming factors POU class 5 homeobox 1 (OCT4), Krueppel-like factor 4 (KLF4), Sex determining region Y-box 2 (SOX2), and Glis Family Zinc Finger 1 (GLIS1). This vector was used to generate induced pluripotent stem (iPS) cells. Here, we applied this SR-RNA vector to generate human iTS cells from aged mesenchymal stem cells (hiTS-M cells) deficient in self-renewal that were derived from adipose tissue. These hiTS-M cells transfected with the SR-RNA vector survived for 15 passages. The hiTS-M cells expressed cell surface markers similar to those of human adipose-derived mesenchymal stem cells (hADSCs) and differentiated into fat cells and osteoblasts. Global gene expression profiling showed that hiTS-M cells were transcriptionally similar to hADSCs. These data suggest that the generation of iTS cells has important implications for the clinical application of autologous stem cell transplantation.
\end{abstract}

Keywords: induced tissue-specific stem (iTS) cells; induced pluripotent stem (iPS) cells; reprogramming factors; adipose-derived mesenchymal stem cells (ADSCs); synthetic self-replicative RNA

\section{Introduction}

Bone marrow-derived mesenchymal stem cells and adipose-derived mesenchymal stem cells (ADSCs) have attracted attention because they have the potential for use in the treatment of various diseases. Numerous published clinical studies have described their use in lower limb revascularization, liver reproduction, cartilage reproduction, and treatment of graft-versus host disease [1-15]. However, the self-renewal competence of ADSCs is limited, and their function diminishes during passage [16-18]. In contrast, embryonic stem (ES) cells and induced pluripotent stem (iPS) cells self-renew and are 
pluripotent because they differentiate into cells derived from the three embryonic germ layers [19-24]. Numerous problems are associated with ES/iPS cell therapy. For example, ES cells may pose ethical issues, and ES and iPS cells may form tumors and have limited in vitro abilities to generate pure populations of differentiated cell types [25]. Therefore, we focused on developing a method for generating induced tissue-specific stem (iTS) cells derived from the pancreas (iTS-P) or liver (ITS-L) by transfecting them with a plasmid that coexpresses the reprogramming factors octamer-binding transcription factor 4 (OCT4), sex-determining region Y-box 2 (SOX2), kruppel-like factor 4 (KLF4), and c-MYC and then performing tissue-specific selection. Notably, the iTS-P/iTS-L cells were unable to generate teratomas when subcutaneously transplanted into immunodeficient mice.

iTS-P and iTS-L cells express genetic markers of pancreatic and hepatic progenitors, respectively. Such cells can be induced to differentiate into insulin-producing cells or hepatocytes, respectively, more frequently than ES cells [26,27]. We generated iTS-P cells using a single synthetic, self-replicating Venezuelan Equine Encephalitis (VEE)-reprogramming factor (RF) RNA replicon (SR-RNA) coexpressing the reprogramming factors OCT4, KLF4, SOX2, and Glis Family Zinc Finger 1 (GLIS1) [28,29].

Here, we applied this SR-RNA vector to generate expandable human iTS cells from aged mesenchymal stem cells (hiTS-M cells) derived from adipose tissue cells deficient in self-renewal.

\section{Results}

\subsection{Generation of hiTS-M Cells from Human ADSCs by an RNA Expression Vector}

We attempted to generate hiTS-M cells from aged human ADSCs (hADSCs) that did not proliferate, cultured for 10 days after the 14th passage (Figure 1D). We transfected the aged hADSCs with the SR-RNA encoding the reprogramming factors OCT4, KLF4, SOX2, and GLIS1 (Figure S1). Aged hADSCs gradually proliferated until four days after transfection with the RNA vector (Figure 1A). Nine days after transfection, the proliferating hiTS-M cells were separately passaged in a new T25 flask. The hiTS-M cells exhibited a fibroblast-like morphology similar to that of hADSCs self-renewed for 15 additional passages, for a total of 29 passages (Figure 1A). In contrast, aged untransfected hADSCs did not proliferate (Figure 1D). Reverse transcription-polymerase chain reaction (RT-PCR) analysis did not detect the SR-RNA in hiTS-M cells at passage five after transfection (total passages: $14+5=19$ ) (Figure 1B). We performed quantitative RT-PCR (qRT-PCR) analysis of marker genes of ES cells to investigate gene expression in hiTS-M cells (passage 29) and hADSCs (passage five). We were unable to detect the mRNAs encoding the pluripotency markers NANOG, OCT4, or SOX2 in either cell type (Figure 1C). We evaluated telomerase activity in hiTS-M cells. Both hADSCs (passage 9) and hiTS-M cells (passage $14+9$ ) did not express human telomerase reverse transcriptase (hTERT) (Figure 1E). 
A

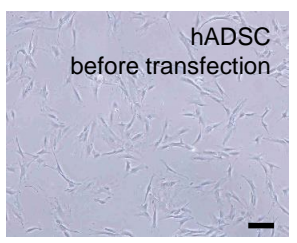

B

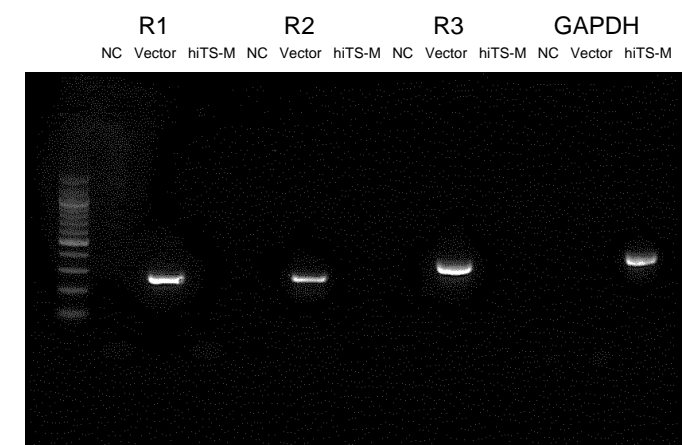

D

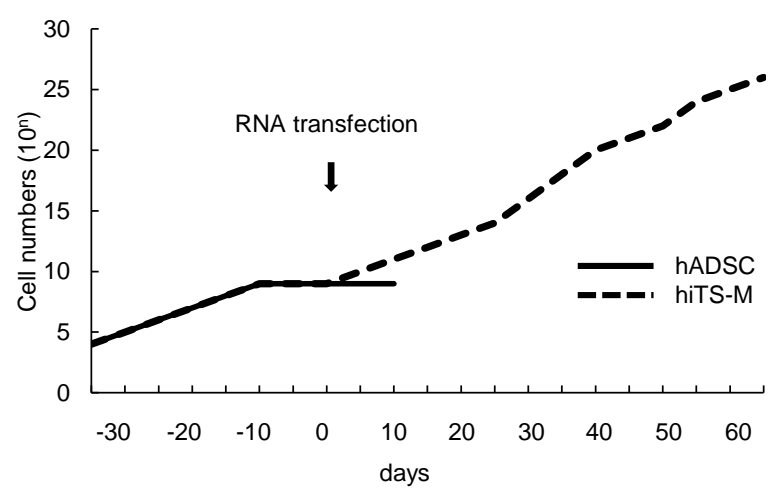

day4

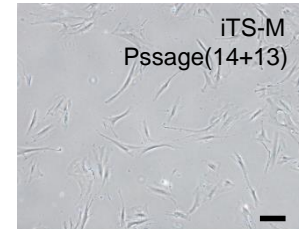

C

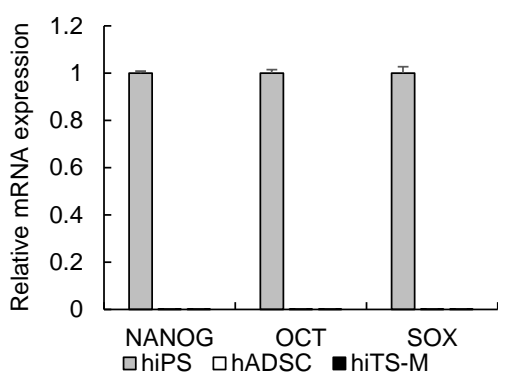

E

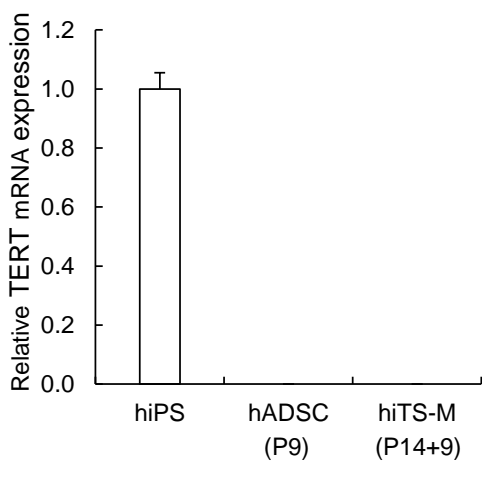

Figure 1. Generation of hiTS-M cells using a synthetic self-replicating RNA. (A) Morphology of hADSCs (passage 14) before transfection, one and four days after transfection, and hiTS-M (passage $14+13$ ). Scale bars $=100 \mu \mathrm{m}$. (B) RT-PCR analysis of persistent VEE-RF RNA Replicon in hiTS-M cells. Total RNA from hiTS-M cells was prepared from passage $(14+5)$. Reactions without template served as negative control (NC). VEE-RF RNA Replicon was used as a positive control (Vec). Locations of R1, R2, and R3 are shown in Figure S1A. Amplicon sizes: R1 = 302 bp, R2 = 302 bp, R3 = 394 bp, GAPDH = $452 \mathrm{bp}$. (C) qRT-PCR analysis of NANOG, OCT4, and SOX2 expression, which are markers of ES/iPS cells, in hiPS cells (passage 20), hADSCs (passage 5), and hiTS-M cells (passage $14+5$ ). Data are expressed as NANOG-, OCT4-, and SOX2-to-GAPDH ratios, with the ratio of iPS cells arbitrarily defined as one $(n=3)$. Error bars represent the standard error. (D) Growth curves of hADSCs (passage 9 to 14) and hiTS-M cells (passage 14 +and 0 to 15). (E) qRT-PCR analysis of hTERT expression in hiPS cells (passage 20), hADSCs (passage 9), and hiTS-M cells (passage $14+9$ ). Data are expressed as $h T E R T$-to-GAPDH ratios, with ratio of iPS cells arbitrarily defined as one $(n=3)$.

\subsection{Characterization of hiTS-M Cells Transfected with the RNA Vector}

We performed flow cytometry to detect cell surface markers characteristic of hADSCs that were expressed by hiTS-M cells. The hiTS-M cells (passage $14+7$ ) and hADSCs (passage 7) expressed integrin $\beta-1$ (CD29) at 99.75\% and 98.37\%, respectively; Thy-1 (CD90) (each 100\%); and hyaluronate receptor/phagocytic glycoprotein-1 (CD44) at 100 and 99.87\%, respectively (Figure 2A-F). The hiTS-M cells and hADSCs rarely expressed protein tyrosine phosphatase, receptor type (CD45) (1.54\% and 2.81\%, 
respectively) and leukocyte common antigen (CD34) (1.74\% and 2.35\%, respectively) (Figure 2G-J). These data suggest that hiTS-M cells expressed hADSC surface markers.

A

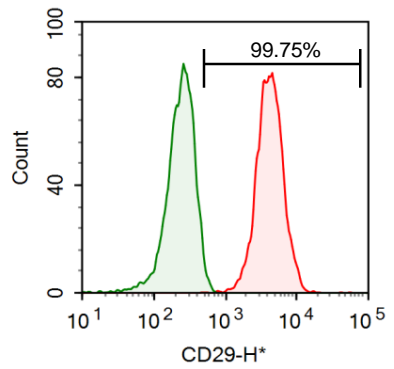

C

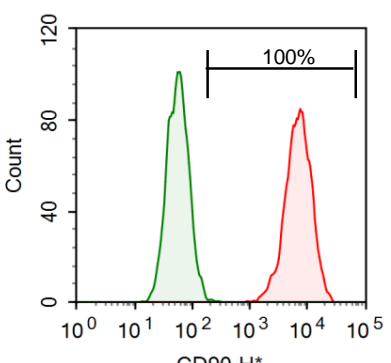

E

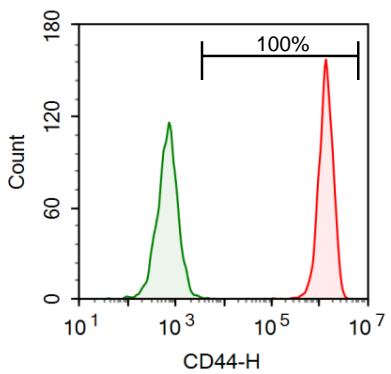

G

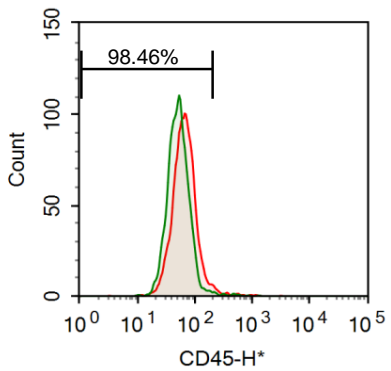

I

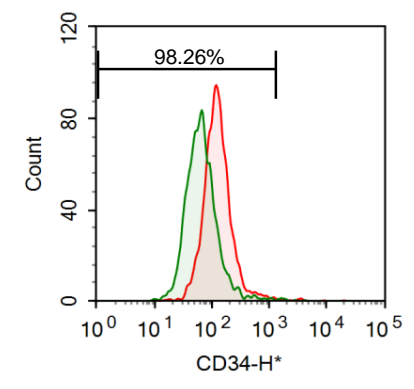

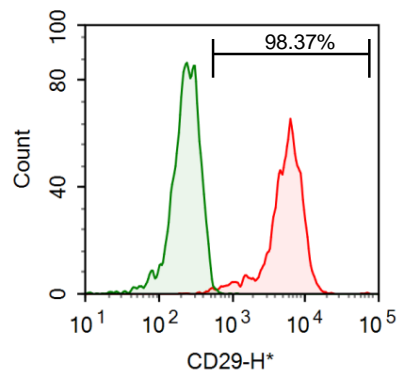

D

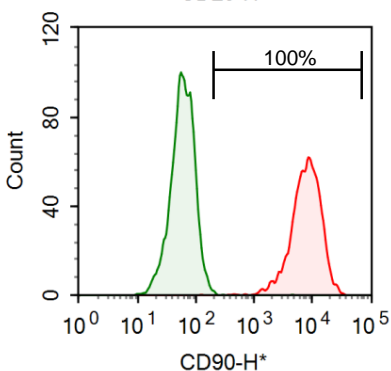

$\mathrm{F}$

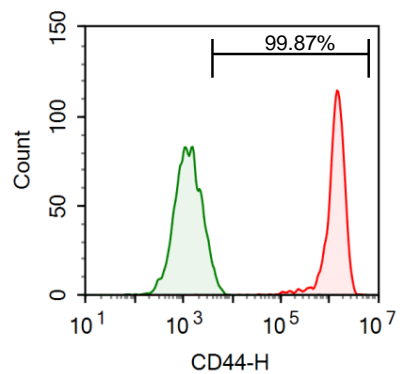

$\mathrm{H}$

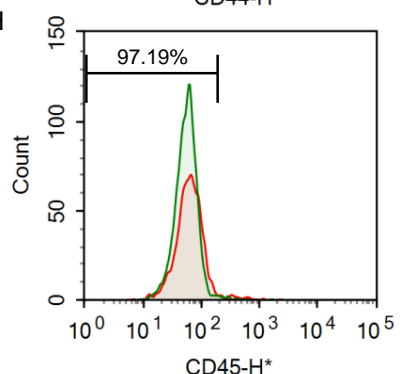

$J$

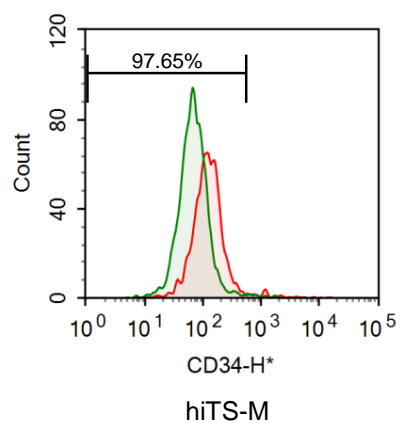

Figure 2. Flow cytometric analysis. hiTS-M cells (passage $14+7$ ) and hADSCs (passage 7) were analyzed: (A) hADSCs, CD29; (B) hiTS-M cells, CD29; (C) hADSCs, CD90; (D) hiTS-M cells, CD90; (E) hADSCs, CD44; (F) hiTS-M cells, CD44; (G) hADSCs, CD45; (H) hiTS-M cells, CD45; (I) hADSCs, CD34; and (J) hiTS-M cells, CD34. 


\subsection{Genes and Proteins Expressed in hiTS-M Cells}

We investigated the mRNAs encoding CD73, CD105, CD55, CD59, CD71, and CD166, which are specific markers for ADSCs. hiTS-M cells (passage 14+6) and hADSCs (passage 6) expressed each mRNA, and the hiTS-M cells expressed significantly higher levels of CD71 mRNA. In contrast, hiTS-M cells expressed significantly lower levels of CD105 and CD55 mRNAs than hADSCs (Figure 3A). hiTS-M cells and hADSCs expressed the mRNAs encoding insulin-like growth factor 1 (IGF1), hepatocyte growth factor (HGF), fibroblast growth factor 2 (FGF2), vascular endothelial cell growth factor A (VEGFA), and epidermal growth factor (EGF). hiTS-M cells expressed HGF and FGF2 at levels four- and six-fold higher compared with hADSCs, respectively. In contrast, hiTS-M cells expressed significantly lower levels of VEGFA and EGF mRNAs compared with hADSCs (Figure 3B).

We also investigated expression of CD73 and CD105 protein by Flow cytometry and immunofluorescence. Both hADSCs and hiTS-M cells expressed CD73 and CD105 protein (Figure 3C,D).

Kumar et al. showed that mesenchymal progenitors derived from human pluripotent stem cells give rise to proliferative pericytes, smooth muscle cells, and mesenchymal stem/stromal cells [9]. We evaluated which cell types hiTS-M cells included. Over $99 \%$ of hiTS-M cells did not express NG2, Calponin, or Desmin, similar to hADSCs (Figure S2). Therefore, over 99\% of hiTS-M cells were mesenchymal stem/stromal cells.

A

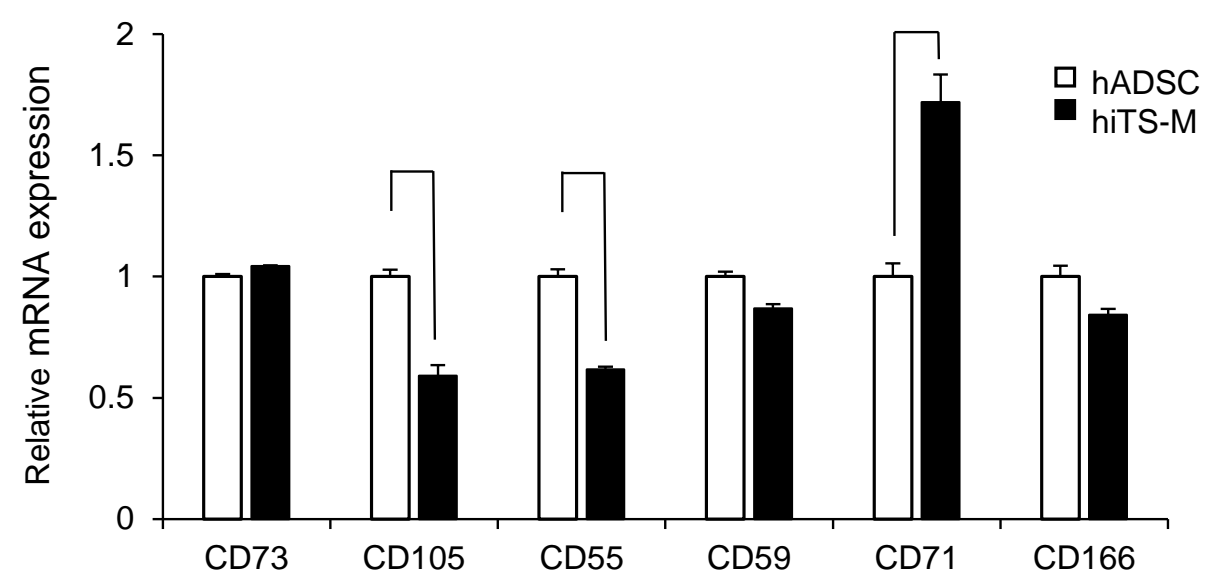

B

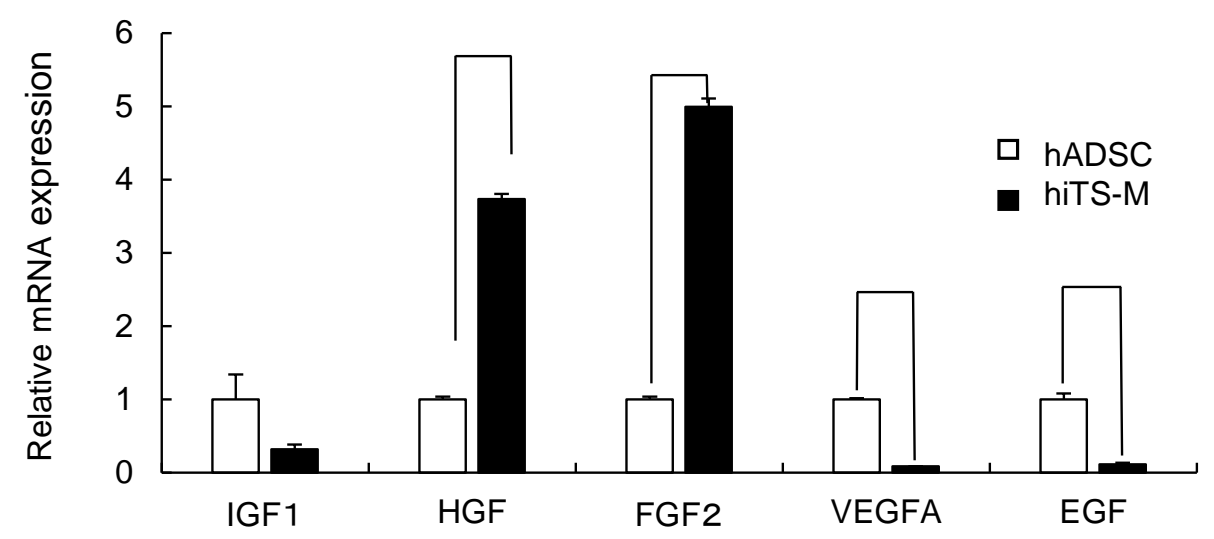

Figure 3. Cont. 
C
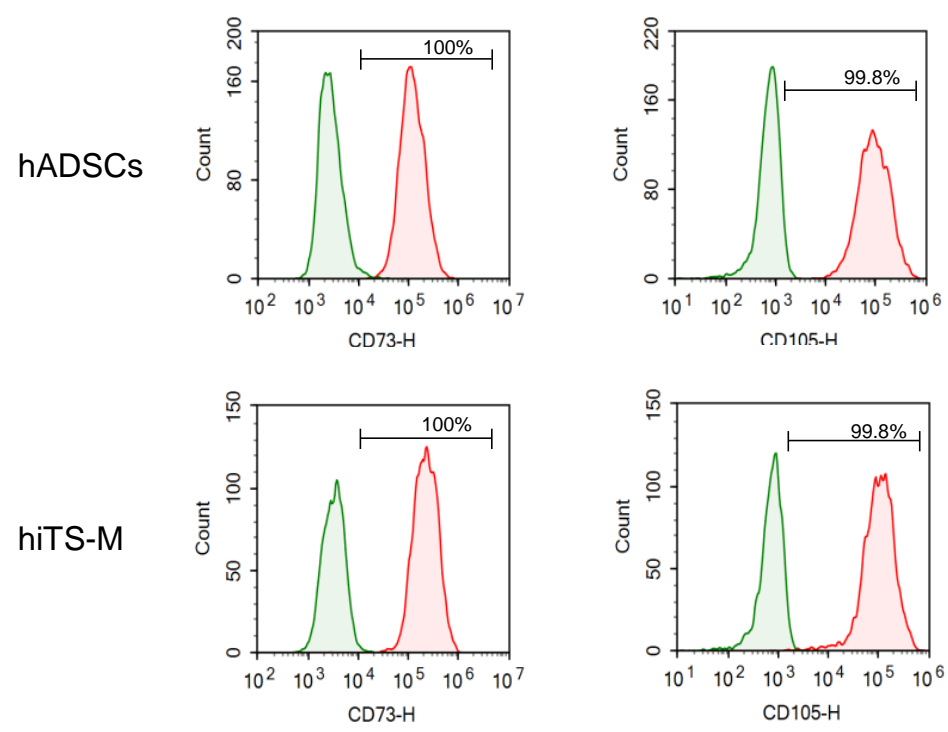

D
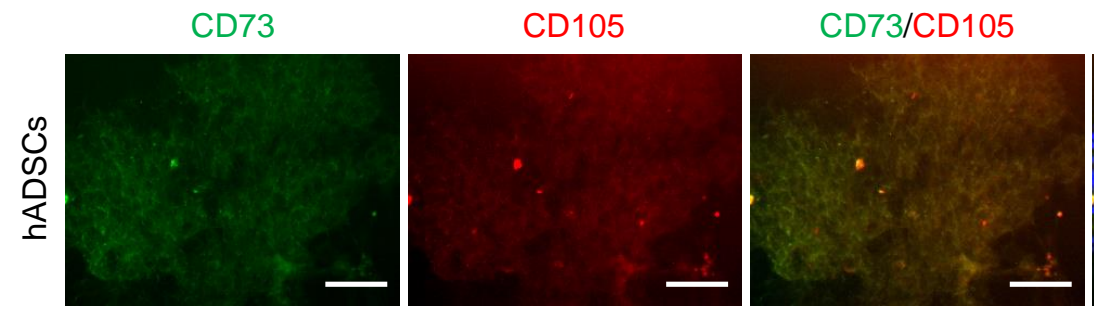

CD73/CD105/DAPI
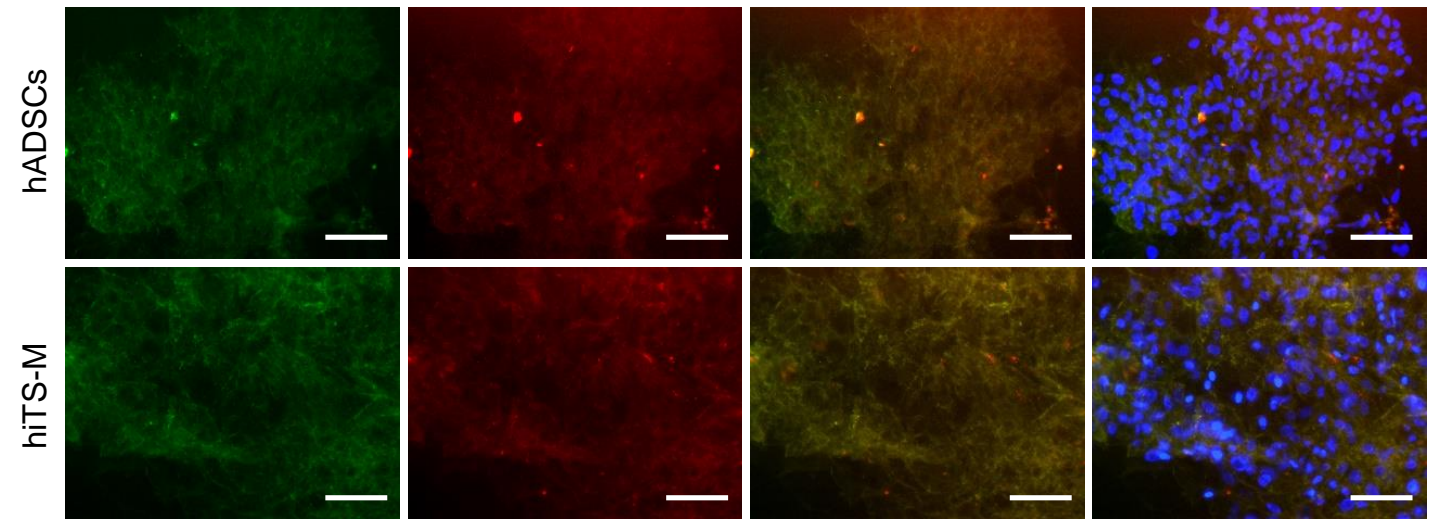

Figure 3. Genes and proteins expressed in hiTS-M cells. (A) qRT-PCR analysis of expression of genes encoding cell surface markers of hiTS-M cells. hADSCs were used as a control. (B) qRT-PCR analysis of expression of marker genes encoding growth factors produced by hiTS-M cells. hADSCs were used as a control. hiTS-M cells (passage $14+7$ ) and hADSCs (passage 7) were used. Data are expressed as mRNA-to-GAPDH mRNA ratio, with the ratio of control cells arbitrarily defined as at one $(n=3)$. Error bars represent the standard error. ${ }^{*} p<0.01$. (C) Flow cytometric analysis of CD73 and CD105. hiTS-M cells (passage $14+7$ ) and hADSCs (passage 7) were analyzed. (D) Immunofluorescence of CD73 and CD105 in hADSCs and hiTS-M cells. Scale bars $=100 \mu \mathrm{m}$.

\subsection{Analysis of the Differentiation Potential of hiTS-M Cells}

To test whether the hiTS-M cells underwent adipogenic differentiation, the cells were treated with adipogenic induction medium for seven days and cultured in maintenance medium for an additional seven days. Oil Red O stained all hiTS-M cells (Figure 4A), suggesting that they exhibited the adipogenic phenotype.

To determine whether the hiTS-M cells underwent osteogenic differentiation, the cells were cultured in osteogenic induction medium for 10 days. All hiTS-M cells possessed calcified nodules revealed by staining with Alizarin Red solution (Figure 4B), suggesting that the treated hiTS-M cells exhibited the osteogenic phenotype. 
A

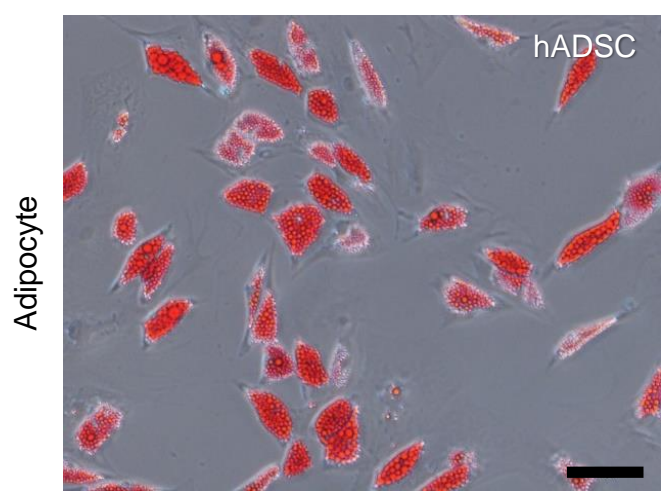

B

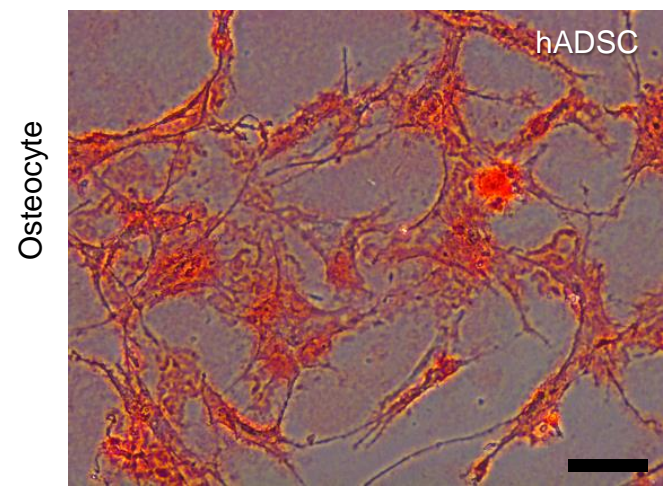

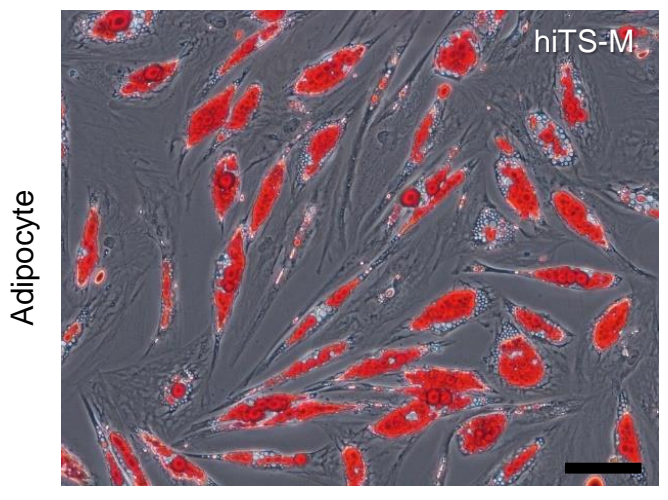

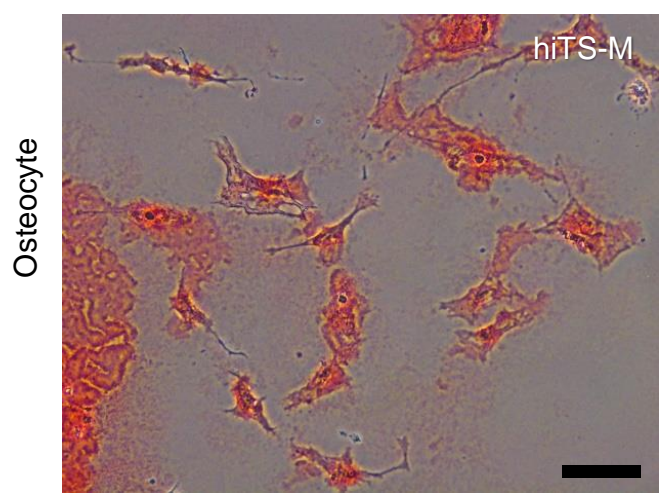

C

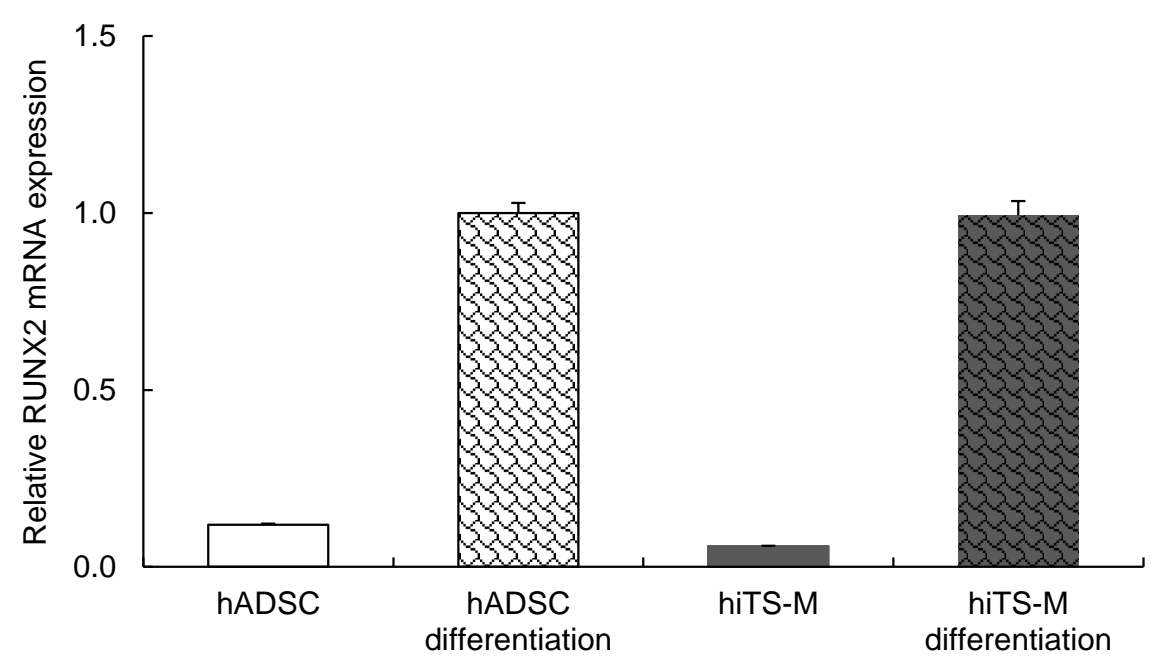

Figure 4. Differentiation of hiTS-M cells into adipocytes and osteocytes. Differentiation of hADSCs and hiTS-M cells into (A) adipocyte and (B) osteocytes. hADSCs (passage 3) and hiTS-M cells (passage $14+4$ ) were used. Scale bars $=100 \mu \mathrm{m}$. (C) qRT-PCR analysis of RUNX2 expression in hADSCs (passage 5) and hiTS-M cells (passage $14+5$ ). Data are expressed as RUNX2-to-GAPDH ratios, with the ratio of differentiated hADSCs arbitrarily defined as one $(n=3)$.

2.5. Bisulfite Sequencing of the Genomic Promoter Regions of OCT4 and NANOG in hiPS Cells, hADSCs, and hiTS-M Cells

Bisulfite genomic sequencing demonstrated that the promoters of OCT4 and NANOG were methylated in hiTS-M cells (passage 14 +6 ) and hADSCs (passage 6). In contrast, these promoters were almost completely demethylated in hiPS cells (passage 20) (Figure 5). These results demonstrate that the extents of methylation of these promoters in hiTS-M cells were similar to those of hADSCs and differed from those of hiPS cells. 


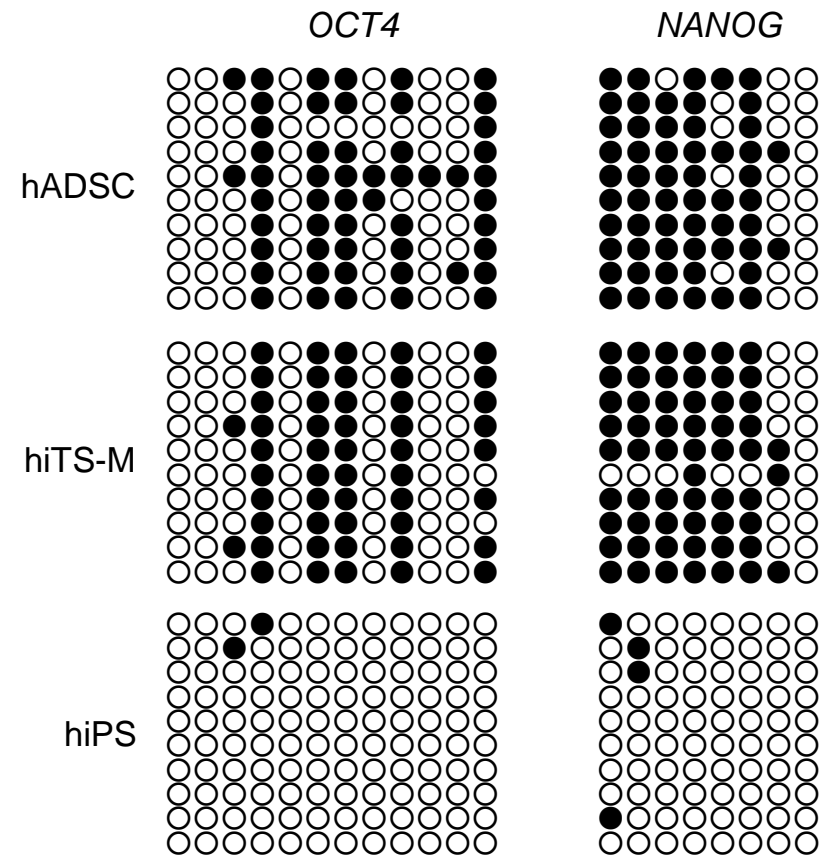

Figure 5. Bisulfite genomic sequencing of promoter regions of OCT4 and NANOG in hADSCs, hiTS-M cells, and hiPS cells. Open circles indicate unmethylated CpG dinucleotides, whereas closed circles indicate methylated CpGs.

\subsection{Microarray Analysis}

We compared the global gene expression profiles of hiPS cells (passage 20), hADSCs (passage 6), and hiTS-M cells (passage $14+6$ ) (Table S3). Of 54,613 genes, $2.13 \%$ differed by more than two-fold between hADSCs and hiTS-M cells; $19.53 \%$ differed by more than two-fold between hiPS cells and hiTS-M cells; and $20.78 \%$ differed by more than two-fold between hiPS cells and hADSCs (Figure 6A). These data suggest that the expression pattern of hiTS-M cells was similar to that of hADSCs but differed from that of hiPS cells. Unsupervised hierarchical clustering of the gene expression profiles of hiTS-M cells, hADSCs, and hiPS cells showed that hiTS-M cells clustered more closely with hADSCs than hiPS cells (Figure 6B).

A

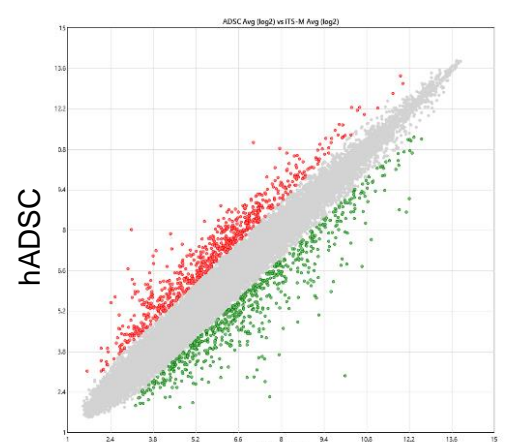

hiTS-M

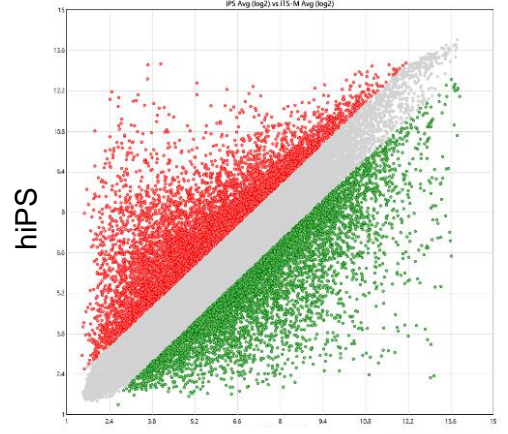

hiTS-M

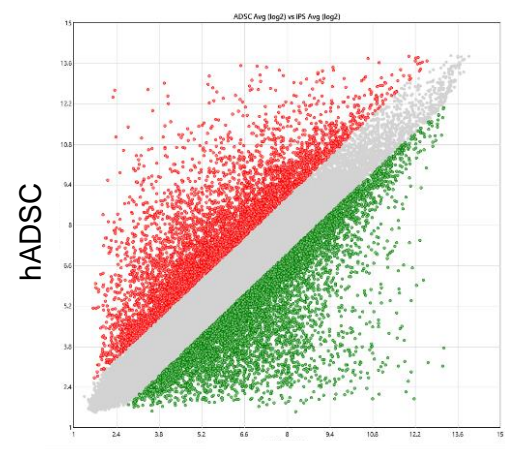

hiPS

Figure 6. Cont. 
B

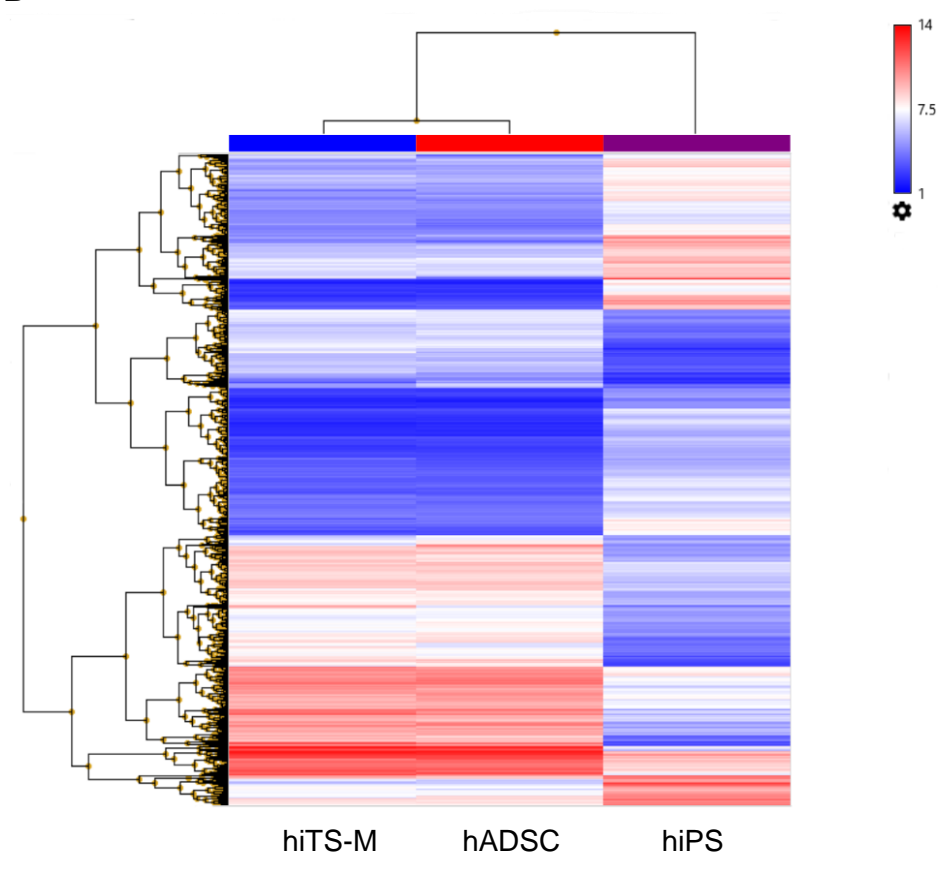

Figure 6. Microarray analysis. (A) A Transcriptome Analysis Console was used to analyze global gene expression patterns between hiPS cells and hiTS-M cells, between hADSCs and hiTS-M cells, and between iPS cells and hADSCs. Grey area indicates genes expressed at levels less than two-fold different between the two samples. (B) Unsupervised hierarchical clustering of gene expression profiles of hiPS cells, hiTS-M cells, and hADSCs. Each column represents one biological sample.

\section{Discussion}

Here, we generated hiTS-M cells using an SR-RNA vector capable of expressing the reprogramming factors OCT4, KLF4, SOX2, and GLIS1. The RNA vector was originally utilized to generate iPS cells [28]. Because hADSC culture medium was used after transfection, iPS cells were not generated in this study. Epigenetic memory is inherited from parental cells following the reprogramming of iPS cells, and the phenotypes of iPS cells may be influenced by the parental cells [30-35]. Although some of the aged hADSCs may have induced hiPS cells immediately after transfection in this study, the cells could not maintain the hiPS cell phenotype because the culture conditions were suitable for propagating hADSCs but not ES/iPS cells. Our present study shows that hiTS-M cells were generated by transient overexpression of reprogramming factors combined with hADSC culture conditions.

ADSCs have a limited self-renewal capacity, and their maximum number of passages is normally $10-15[36,37]$. We showed here that the hADSCs could be passaged 15 times after transfection (14 passages before transfection and 15 passages after transfection), and the cell number increased from $10^{9}$ (day 0) to $10^{26}$ (day 70) (Figure 1D). Extending the passage number is important for autologous transplantation of ADSCs. In contrast, the characteristics of hiTS-M cells were similar but not identical to those of hADSCs as indicated by qRT-PCR and microarray analyses. The levels of mRNAs encoding CD105, CD55, VEGFA, and EGF were lower in hiTS-M cells compared with those of hADSCs, whereas those of the mRNAs encoding CD71, HGF, and FGF2 were higher in hiTS-M cells compared with those of hADSCs. This may be explained by the degree of reprogramming induced by the proteins expressed by the SR-RNA vector.

In this study, we generated human iTS-M cells using a single, synthetic, self-replicating VEE-RF RNA replicon. Other groups, including ours, have used plasmid transfection to generate iPS/iTS cells $[26,27]$. However, these showed a DNA integration of the plasmid of around $10-20 \%$. RNA vector was used in this study to avoid potential integration problems. 
The characteristics of ADSCs change during passage [36,37]. When aged hADSCs are strongly reprogrammed by the high expression of reprogramming factors, the characteristics of the hiTS-M cells may be similar to those of hADSCs during early passages. When aged hADSCs are weakly reprogrammed by the low expression of reprogramming factors, the characteristics of the hiTS-M cells may be similar to those of late passage hADSCs. The characteristics of hiTS-M cells may depend on the degree of reprogramming and their passage number after transfection. As shown in Figure 4, both hiTS-M and hADSCs appear to have partially lost the ability to differentiate. This may be due to the variability in hADSCs and in the reprogramming of hiTS-M cells.

In conclusion, we generated hiTS-M cells from aged hADSCs. hiTS-M cells expressed cell surface markers similar to those of hADSCs and were induced to differentiate into fat cells and osteoblasts. qRT-PCR and bisulfite genomic sequencing analyses of OCT4 and NANOG showed that the phenotype of hiTS-M cells differed significantly from that of hiPS cells. Global gene-expression profiling showed that hiTS-M cells are transcriptionally similar to hADSCs. Because the self-renewal capacity of hADSCs is restricted and the function of hADSCs decreases during passage, the generation of hiTS-M cells may have important implications for the clinical application of autologous stem cell transplantation.

\section{Materials and Methods}

\subsection{Culture of $h A D S C s$}

Human ADSCs derived from a 40-year-old female donor (Lot No. 0000353102; body mass index, 23; passage 1) were obtained from Lonza Japan, Inc. (Tokyo, Japan). For adherent cell culture, $4 \times 10^{5}$ hADSC s were seeded into a T-25 flask (Asone, Osaka, Japan) using the Poietics ADSC-GM BulletKit (PT-4505; Lonza Japan) at $37^{\circ} \mathrm{C}$ in a humidified atmosphere containing $5 \% \mathrm{CO}_{2}$. The cells were cultured until they were approximately $80-90 \%$ confluent. The cells were detached from the culture flask using trypsin-ethylenediaminetetraacetic acid (EDTA) $(0.25 \% w / v$; Wako, Osaka, Japan) and repeatedly seeded and cultured in new culture flasks. The hADSCs were passaged 14 times until they failed to proliferate. The hADSCs, from passages four to seven, were used as a control for the analyses as follows: RT-PCR, qRT-PCR, differentiation into adipocytes and osteocytes, bisulfite genomic sequencing, and microarray analysis.

\subsection{Generation of hiTS-M Cells Using an RNA Replicon}

The hiTS-M cells were generated as described previously [28], with minor modification, using a Simplicom RNA Reprogramming Kit (Merck Millipore, Tokyo, Japan). Aged hADSCs (passage 14) were seeded into a T25 plate on day-10 (40-50\% confluency). To minimize the interferon response, cells were treated with $1 \mathrm{~mL}$ of Advanced DMEM (Thermo Fisher Scientific, Waltham, MA, USA) containing $0.2 \mu \mathrm{g}$ of B18R protein (Merck Millipore, Burlington, MA, USA) $2 \mathrm{~h}$ before transfection. On day 0, $0.5 \mu \mathrm{g}$ VEE-OKS-iG and $0.5 \mu \mathrm{g}$ B18R mRNAs was used to transfect cells in the presence of Lipofectamine 2000 (Thermo Fisher Scientific). After $3 \mathrm{~h}$, the transfection medium was changed to Advanced DMEM containing $200 \mathrm{ng} / \mathrm{mL}$ of B18R protein. The cells were passaged on day 9, and Advanced DMEM containing $200 \mathrm{ng} / \mathrm{mL}$ of B18R protein was supplied every day until day 16 . Cells were then passaged on day 16 and Advanced DMEM was replaced with hADSC culture medium (Figure S1).

\subsection{RT-PCR and $q R T-P C R$ Analyses}

Total RNA was extracted from cells using an RNeasy Mini Kit (Qiagen, Tokyo, Japan). After quantifying the RNA using spectrophotometry, $2.5 \mu \mathrm{g}$ RNA was heated at $85{ }^{\circ} \mathrm{C}$ for $3 \mathrm{~min}$ and then reverse-transcribed in a $25-\mu \mathrm{L}$ solution containing 200 units of Superscript II RNase H-RT (Thermo Fisher Scientific), 50 ng random hexamers (Thermo Fisher Scientific), $160 \mu \mathrm{mol} / \mathrm{L}$ dNTP, and $10 \mathrm{nmol} / \mathrm{L}$ dithiothreitol. The reaction conditions were as follows: $10 \mathrm{~min}$ at $25^{\circ} \mathrm{C}, 60 \mathrm{~min}$ at $42{ }^{\circ} \mathrm{C}$, and $10 \mathrm{~min}$ at $95^{\circ} \mathrm{C}$. Amplifications were performed using a Perkin-Elmer 9700 Thermocycler (Perkin-Elmer, Waltham, CT, USA) with $3 \mu \mathrm{L}$ of cDNA (20 ng DNA equivalents), $160 \mu \mathrm{mol} / \mathrm{L}$ of 
cold dNTPs, 10 pmol of appropriate oligonucleotide primers, $1.5 \mathrm{mmol} / \mathrm{L}$ of $\mathrm{MgCl}_{2}$, and 5 units of AmpliTaq Gold DNA polymerase (Perkin-Elmer, Norwalk, CT, USA) in $1 \times$ PCR buffer. The thermal cycle profile was as follows: $10 \mathrm{~min}$ denaturation at $94^{\circ} \mathrm{C}$ followed by an amplification cycle $(1 \mathrm{~min}$ denaturation at $94{ }^{\circ} \mathrm{C}, 1 \mathrm{~min}$ annealing at $57^{\circ} \mathrm{C}$ to $62^{\circ} \mathrm{C}$, and a 1 min extension at $72{ }^{\circ} \mathrm{C}$ ) with a final extension step of $10 \mathrm{~min}$ at $72{ }^{\circ} \mathrm{C}$. The oligonucleotide primers are shown in Table S1.

The mRNAs were quantified using a TaqMan Real-time PCR System (Applied Biosystems, Foster City, CA, USA) according to the manufacturer's instructions. PCR was performed for 40 cycles, including $2 \mathrm{~min}$ at $50{ }^{\circ} \mathrm{C}$ and $10 \mathrm{~min}$ at $95^{\circ} \mathrm{C}$ as initial steps. In each cycle, denaturation was performed for $15 \mathrm{~s}$ at $95^{\circ} \mathrm{C}$, and annealing and extension were performed for $1 \mathrm{~min}$ each at $60^{\circ} \mathrm{C}$. PCR was performed in $20 \mu \mathrm{L}$ of a solution of cDNAs synthesized from $1.11 \mathrm{ng}$ of total RNA. The level of each mRNA sample was normalized by dividing its amount by that of GAPDH mRNA. Primers for the mRNAs encoding OCT4, SOX2, NANOG, TERT, 5'-nucleotidase (CD73), endoglin (CD105), complement decay accelerating factor (CD55), complement protectin (CD59), transferrin receptor (CD71), and activated lymphocyte cell adhesion molecule (CD166); primers specific for the genes encoding IGF1, HGF, FGF2, VEGFA, EGF, RUNX2, and GAPDH were included in Assays-on-Demand Gene Expression Products (Thermo Fisher Scientific).

\subsection{Flow Cytometry}

Flow cytometry was performed using a NovoCyte Flow Cytometer (ACEA Biosciences, Inc., San Diego, CA, USA) according to the manufacturer's instructions. Briefly, hADSCs $\left(1 \times 10^{5}\right.$ cells $)$ were suspended in $0.5 \mathrm{~mL}$ phosphate-buffered saline (PBS) (Wako, Osaka, Japan). Each antibody (1/100 volume) was added to the cell suspensions and incubated on ice for $30 \mathrm{~min}$. Analyses were performed after washing the cells with Brilliant Stain Buffer (BD Biosciences, Franklin Lakes, NJ, USA). Primary antibodies were as follows: Allophycocyanin (APC) Mouse Anti-Human, CD29, BV421 Mouse Anti-Human CD44, BV421 Mouse IgG2b k Isotype Control, APC Mouse IgG1 k Isotype Control (BD Biosciences); FITC anti-human CD90.2 (Thy1) Antibody, FITC Mouse IgG1 k Isotype Ctrl Antibody, PerCP anti-human CD34 Antibody, PerCP Mouse IgG1 k Isotype Ctrl Antibody, PE/Cy7 anti-human CD45 Antibody, PE/Cy7 Mouse IgG1 к Isotype Ctrl Antibody, FITC anti-human CD73 (Ecto-5'-nucleotidase) Antibody, and Alexa Fluor 647 anti-human CD105 Antibody (BioLegend, Inc., San Diego, CA, USA) [38].

\subsection{Immunostaining}

Cell were fixed with $4 \%$ paraformaldehyde in PBS buffer. After blocking with 20\% AquaBlock (EastCoast Bio, Inc. North Berwick, ME, USA) for $30 \mathrm{~min}$ at room temperature, the cells were incubated overnight at $4{ }^{\circ} \mathrm{C}$ with each 1st antibody (FITC anti-human CD73 (Ecto-5'-nucleotidase) Antibody, Alexa Fluor 647 anti-human CD105 Antibody, Alexa Fluor 488 anti-human NG2 Antibody, mouse anti-human Calponin Antibody, and rabbit anti-Desmin Antibody) and then for $1 \mathrm{~h}$ at room temperature with each second antibody (AlexaFluor 488 anti-mouse antibody, AlexaFluor 488 anti-rabbit antibody). Mounting medium for fluorescence with DAPI (Vector Laboratories Inc., Burlingame, CA, USA) was used for mounting.

\subsection{Cell Differentiation}

Adipogenic differentiation was performed using Adipogenic Differentiation Medium (DM-2; Zen-Bio, Inc., Research Triangle Park, NC, USA) and Adipocyte Maintenance Medium (AM-1; Zen-Bio, Inc.). Confluent hiTS-M cells (passage $14+7$ ) and hADSCs (passage 7) (three to four days after seeding into six-well plates using the designated medium) were cultured for seven days using Adipocyte Differentiation Medium (DM-2; Zen-Bio, Inc.) to induce differentiation into adipocytes. The medium was subsequently switched to Adipocyte Maintenance Medium (AM-1; Zen-Bio, Inc.) and changed every three days. Adipocytes with lipid droplets were detected using light microscopy after 20-30 days. Differentiation was confirmed by Oil Red O (Cosmo Bio Co., Ltd., Tokyo, Japan) 
staining of intracellular lipid droplets. Differentiated ASCs were fixed in $10 \%$ formaldehyde (Wako, Osaka, Japan) in PBS (Wako, Osaka, Japan) for at least 10 min, washed with 60\% isopropanol (Wako, Osaka, Japan), and stained with Oil Red O (Wako, Osaka, Japan) for $10 \mathrm{~min}$ followed by repeated washing with water and destaining in 100\% isopropanol for 1 min $[14,25,39]$.

Osteogenic differentiation was induced by culturing the hiTS-M cells and hADSCs (passage 3) for 10 days in MSC Go Rapid Osteogenic XF ${ }^{\mathrm{TM}}$ (Cosmo Bio Co., Ltd., Tokyo, Japan). Differentiation was determined through detection of extracellular matrix calcification as revealed by staining with Alizarin Red solution (ARD-A1; PG Research Co., Ltd., Tokyo, Japan) according to the manufacturer's instructions $[10,14,25,39,40]$.

\subsection{Bisulfite Genomic Sequencing}

Bisulfite treatment was performed using the CpGenome Turbo Bisulfite Modification Kit (Merck Millipore) according to the manufacturer's recommendations. The PCR primers are listed in Table S2. Amplicons were cloned using a Mighty TA-cloning kit (TAKARA BIO INC., Shiga, Japan). Ten randomlyselected clones were sequenced using M13 forward and reverse primers specific for each gene.

\subsection{Microarray Analysis}

Total RNA from ES cells, iTS-P cells, or islets was labeled with biotin. Samples were hybridized to the GeneChip 3'IVT PLUS Reagent Kit (Affymetrix, Tokyo, Japan) and GeneChip Hybridization, Wash and Stain Kit (Affymetrix, Tokyo, Japan) according to the manufacturer's protocol. Arrays were scanned using a GeneChip Scanner 3000 7G (Affymetrix, Tokyo, Japan). Data were analyzed using Transcriptome Analysis Console 4.0 software (Thermo Fisher).

\subsection{Statistical Analyses}

The data are expressed as the mean \pm standard error. Two groups were compared using the Student $t$-test. The differences between each group were considered significant if $p<0.01$. All statistical analysis methods were performed in accordance with the relevant guidelines and regulations.

Supplementary Materials: The following are available online at http:/ /www.mdpi.com/1422-0067/19/11/3489/s1.

Author Contributions: H.N. designed the experiments and analyzed the data. C.M.-S. performed most of the experimental work with the help of Y.N., N.K., S.K., I.S. and M.W. provided materials and discussion. C.M.-S. and H.N. wrote the manuscript. All authors discussed and commented on the manuscript.

Funding: This work was supported in part by JSPS KAKENHI Grant Numbers JP16H05404, JP16K10435, JP18K08545; Japan Agency for Medical Research and Development, Okinawa Science and the Technology Innovation System Construction Project, the Waksman Foundation of Japan, Inc., and The Naito Foundation.

Acknowledgments: We thank Naomi Kakazu (University of the Ryukyus) for administrative assistance and Maki Higa, Yuki Kawahira, and Saori Adaniya (University of the Ryukyus) for technical support.

Conflicts of Interest: The authors declare no conflict of interest.

\section{References}

1. Friedenstein, A.J.; Petrakova, K.V.; Kurolesova, A.I.; Frolova, G.P. Heterotopic of bone marrow. Analysis of precursor cells for osteogenic and hematopoietic tissues. Transplantation 1968, 6, 230. [CrossRef] [PubMed]

2. Zuk, P.A. Human Adipose Tissue Is a Souce of Multipotent Stem Cells. Mol. Biol. Cell 2002, 13, 4279-4295. [CrossRef] [PubMed]

3. Dominici, M.; Le Blanc, K.; Mueller, I.; Slaper-Cortenbach, I.; Marini, F.; Krause; Deans, R.; Keating, A.; Prockop, D.J.; Horwitz, E. Minimal criteria for defining multipotent mesenchymal stromal cells. The International Society for Cellular Therapy position statement. Cytotherapy 2006, 8, 315-317. [CrossRef] [PubMed]

4. Ohgushi, H.; Caplan, A.I. Stem cell technology and bioceramics: From cell to gene engineering. J. Biomed. Mater. Res. 1999, 48, 913-927. [CrossRef]

5. Caplan, A.I. Mesenchymal stem cells. J. Orthop. Res. 1991, 9, 641-650. [CrossRef] [PubMed] 
6. King, S.N.; Hanson, S.E.; Hematti, P.; Thibeault, S.L. Current applications of mesenchymal stem cells for tissue replacement in otolaryngology-head and neck surgery. Am. J. Stem Cells 2012, 1, 225-238. [PubMed]

7. Mathe, G.; Bernard, J.; Schwarzenberg, L.; Larriue, M.J.; Lalanne, C.M.; Dutrieux, A.; Denoix, P.F.; Sur-mont, J.; Schwarzmann, V.; Ceoara, B. Trial treatment of patients afflicted with acute leukemia in remission with total irradiation followed by homologous bone marrow transfusion. Rev. Fr. Etud. Clin. Biol. 1959, 4, 675-704. [PubMed]

8. Mathe, G.; Jammet, H.; Pendic, B.; Schwarzenberg, L.; Duplan, J.F.; Maupin, B.; Lataret, R.; Larriue, M.J.; Kalic, D.; Djukic, Z. Transfusion and grafts of homologous bonemarrow in humans after accidental high dose irradiation. Rev. Fr. Etud. Clin. Biol. 1959, 4, 226-238. [PubMed]

9. Kumar, A.; D’Souza, S.S.; Moskvin, O.V.; Toh, H.; Wang, B.; Zhang, J.; Swanson, S.; Guo, L.W.; Thomson, J.A.; Slukvin, I.I. Specification and Diversification of Pericytes and Smooth Muscle Cells from Mesenchymoangioblasts. Cell Rep. 2017, 19, 1902-1916. [CrossRef] [PubMed]

10. Kumar, A.; Salimath, B.P.; Schieker, M.; Stark, G.B.; Finkenzeller, G. Inhibition of metastasis-associated gene 1 expression affects proliferation and osteogenic differentiation of immortalized human mesenchymal stem cells. Cell Prolif. 2011, 44, 128-138. [CrossRef] [PubMed]

11. Gaur, M.; Dobke, M.; Lunyak, V.V. Mesenchymal Stem Cells from Adipose Tissue in Clinical Applications for Dermatological Indications and Skin Aging. Int. J. Mol. Sci. 2017, 18, 208. [CrossRef] [PubMed]

12. Iwazawa, R.; Kozakai, S.; Kitahashi, T.; Nakamura, K.; Hata, K.I. The Therapeutic Effects of Adipose-Derived Stem Cells and Recombinant Peptide Pieces on Mouse Model of DSS Colitis. Cell Transpl. 2018, 27, 1390-1400. [CrossRef] [PubMed]

13. Tabatabaei Qomi, R.; Sheykhhasan, M. Adipose-derived stromal cell in regenerative medicine: A review. World J. Stem Cells 2017, 9, 107-117. [CrossRef] [PubMed]

14. Owczarczyk-Saczonek, A.; Wociór, A.; Placek, W.; Maksymowicz, W.; Wojtkiewicz, J. The Use of AdiposeDerived Stem Cells in Selected Skin Diseases (Vitiligo, Alopecia, and Nonhealing Wounds). Stem Cells Int. 2017, 2017, 4740709. [CrossRef] [PubMed]

15. Nagaya, N.; Fujii, T.; Iwase, T.; Ohgushi, H.; Itoh, T.; Uematsu, M.; Yamagishi, M.; Mori, H.; Kanagawa, K.; Kitamura, S. Intravenous administration of mesenchymal stem cells improves cardiac function in rats with acute myocardial infarction through angiogenesis and myogenesis. Am. J. Physiol. Heart Circ. Physiol. 2004, 287, 2670-2676. [CrossRef] [PubMed]

16. Patrick, C.W., Jr. Adipose tissue engineering: The future of breast and soft tissue reconstruction following tumor resection. Semin. Surg. Oncol. 2000, 19, 302-311. [CrossRef]

17. Patrick, C.W. Breast tissue engineering. Annu. Rev. Biommed. Eng. 2004, 6, 109-130. [CrossRef] [PubMed]

18. Beahm, E.K.; Walton, R.L.; Patrick, C.W., Jr. Progress in adipose tissue construct development. Clin. Plast. Surg. 2003, 30, 547-558. [CrossRef]

19. Takahashi, K.; Yamanaka, S. Induction of pluripotent stem cells from mouse embryonic and adult fibroblast cultures by defined factors. Cell 2006, 126, 663-676. [CrossRef] [PubMed]

20. Takahashi, K.; Tanabe, K.; Ohnuki, M.; Narita, M.; Ichisaka, T.; Tomoda, K.; Yamanaka, S. Induction of pluripotent stem cells from adult human fibroblasts by defined factors. Cell 2007, 131, 861-872. [CrossRef] [PubMed]

21. Park, I.H.; Zhao, R.; West, J.A.; Yabuuchi, A.; Huo, H.; Ince, T.A.; Lensch, M.W.; Daley, G.Q. Reprogramming of human somatic cells to pluripotency with defined factors. Nature 2008, 451, 141-146. [CrossRef] [PubMed]

22. Maherali, N.; Sridharan, R.; Xie, W.; Utikal, J.; Eminli, S.; Arnold, K.; Stadtfeld, M.; Yachechko, R.; Tchieu, J.; Jaenisch, R.; et al. Directly reprogrammed fibroblasts show global epigenetic remodeling and widespread tissue contribution. Cell Stem Cell 2007, 1, 55-70. [CrossRef] [PubMed]

23. Okita, K.; Ichisaka, T.; Yamanaka, S. Generation of germline-competent induced pluripotent stem cells. Nature 2007, 448, 313-317. [CrossRef] [PubMed]

24. Wernig, M.; Meissner, A.; Foreman, R.; Brambrink, T.; Ku, M.; Hochedlinger, K.; Bernstein, B.E.; Jaenisch, R. In vitro reprogramming of fibroblasts into a pluripotent ES-cell-like state. Nature 2007, 448, 318-324. [CrossRef] [PubMed]

25. Miyagi-Shiohira, C.; Nakashima, Y.; Kobayashi, N.; Saitoh, I.; Watanabe, M.; Noguchi, Y.; Kinjo, T.; Noguchi, H. The Development of Cancer through the Transient Overexpression of Reprogramming Factors. Cell Med. 2018, 10, 1-7. [CrossRef] 
26. Noguchi, H.; Saitoh, I.; Tsugata, T.; Kataoka, H.; Watanabe, M.; Noguchi, Y. Induction of tissue-specific stem cells by reprogramming factors, and tissue-specific selection. Cell Death Differ. 2015, 22, 145-155. [CrossRef] [PubMed]

27. Saitoh, I.; Sato, M.; Soda, M.; Inada, E.; Iwase, Y.; Murakami, T.; Ohshima, H.; Hayasaki, H.; Noguchi, H. Tissue-Specific Stem Cells Obtained by Reprogramming of Non-Obese Diabetic (NOD) Mouse-Derived Pancreatic Cells Confer Insulin Production in Response to Glucose. PLoS ONE 2016, 11, e0163580. [CrossRef] [PubMed]

28. Yoshioka, N.; Gros, E.; Li, H.R.; Kumar, S.; Deacon, D.C.; Maron, C.; Muotri, A.R.; Chi, N.C.; Fu, X.D.; $\mathrm{Yu}$, B.D.; et al. Efficient generation of human iPSCs by a synthetic self-replicative RNA. Cell Stem Cell 2013, 13, 246-254. [CrossRef] [PubMed]

29. Miyagi-Shiohira, C.; Nakashima, Y.; Kobayashi, N.; Saitoh, I.; Watanabe, M.; Noguchi, H. Characterization of induced tissue-specific stem cells from pancreas by a synthetic self-replicative RNA. Sci. Rep. 2018, 8, 12341. [CrossRef] [PubMed]

30. Kim, K.; Doi, A.; Wen, B.; Ng, K.; Zhao, R.; Cahan, P.; Kim, J.; Aryee, M.J.; Ji, H.; Ehrlich, L.I.; et al. Epigenetic memory in induced pluripotent stem cells. Nature 2010, 467, 285-290. [CrossRef] [PubMed]

31. Polo, J.M.; Liu, S.; Figueroa, M.E.; Kulalert, W.; Eminli, S.; Tan, K.Y.; Apostolou, E.; Stadtfeld, M.; Li, Y.; Shioda, T.; et al. Cell type of origin influences the molecular and functional properties of mouse induced pluripotent stem cells. Nat. Biotechnol. 2010, 28, 848-855. [CrossRef] [PubMed]

32. Lister, R.; Pelizzola, M.; Kida, Y.S.; Hawkins, R.D.; Nery, J.R.; Hon, G.; Antosiewicz-Bourget, J.; O’Malley, R.; Castanon, R.; Klugman, S.; et al. Hotspots of aberrant epigenomic reprogramming in human induced pluripotent stem cells. Nature 2011, 471, 68-73. [CrossRef] [PubMed]

33. Doi, A.; Park, I.H.; Wen, B.; Murakami, P.; Aryee, M.J.; Irizarry, R.; Herb, B.; Ladd-Acosta, C.; Rho, J.; Loewer, S.; et al. Differential methylation of tissue- and cancer-specific CpG island shores distinguishes human induced pluripotent stem cells, embryonic stem cells and fibroblasts. Nat. Genet. 2009, 41, 1350-1353. [CrossRef] [PubMed]

34. Ohi, Y.; Qin, H.; Hong, C.; Blouin, L.; Polo, J.M.; Guo, T.; Qi, Z.; Downey, S.L.; Manos, P.D.; Rossi, D.J.; et al. Incomplete DNA methylation underlies a transcriptional memory of somatic cells in human iPS cells. Nat. Cell Biol. 2011, 13, 541-549. [CrossRef] [PubMed]

35. Noguchi, H.; Miyagi-Shiohira, C.; Nakashima, Y. Induced Tissue-Specific Stem Cells and Epigenetic Memory in Induced Pluripotent Stem Cells. Int. J. Mol. Sci. 2018, 19, 930. [CrossRef] [PubMed]

36. Panasophonkul, S.; Samart, P.; Kongon, K.; Sathanawongs, A. Phenotypic characteristics of feline adipose-derived stem cells affected by cell passage number. Pol. J. Vet. Sci. 2017, 20, 651-660. [CrossRef] [PubMed]

37. Legzdina, D.; Romanauska, A.; Nikulshin, S.; Kozlovska, T.; Berzins, U. Characterization of Senescence of Culture-expanded Human Adipose-derived Mesenchymal Stem Cells. Int. J. Stem Cells 2016, 9, 124-136. [CrossRef] [PubMed]

38. Nakashima, Y.; Nahar, S.; Miyagi-Shiohira, C.; Kinjo, T.; Kobayashi, N.; Saitoh, I.; Watanabe, M.; Fujita, J.; Noguchi, H. A Liquid Chromatography with Tandem Mass Spectrometry-Based Proteomic Analysis of Cells Cultured in DMEM 10\% FBS and Chemically Defined Medium Using Human Adipose-Derived Mesenchymal Stem Cells. Int. J. Mol. Sci. 2018, 19, 2042. [CrossRef] [PubMed]

39. Miyagi-Shiohira, C.; Nakashima, Y.; Kobayashi, N.; Saitoh, I.; Watanabe, M.; Noguchi, H. Evaluation of Serum-Free, Xeno-Free Cryopreservation Solutions for Human Adipose-Derived Mesenchymal Stem Cells. Cell Med. 2016, 9, 15-20. [CrossRef] [PubMed]

40. Sugimoto, A.; Miyazaki, A.; Kawarabayashi, K.; Shono, M.; Akazawa, Y.; Hasegawa, T.; Ueda-Yamaguchi, K.; Kitamura, T.; Yoshizaki, K.; Fukumoto, S.; et al. Piezo type mechanosensitive ion channel component 1 functions as a regulator of the cell fate determination of mesenchymal stem cells. Sci. Rep. 2017, 7, 17696. [CrossRef] [PubMed]

(c) 2018 by the authors. Licensee MDPI, Basel, Switzerland. This article is an open access article distributed under the terms and conditions of the Creative Commons Attribution (CC BY) license (http:/ / creativecommons.org/licenses/by/4.0/). 